\title{
Le B.B. : un outil de la formation d'une communauté savante
}

Danièle Hervieu-Leger

\section{(2) OpenEdition \\ 12 Journals}

Édition électronique

URL : http://journals.openedition.org/assr/3815

DOI : $10.4000 /$ assr.3815

ISSN : $1777-5825$

Éditeur

Éditions de l'EHESS

Édition imprimée

Date de publication : 1 décembre 2006

Pagination : 53-61

ISBN : 2-7132-2124-2

ISSN : 0335-5985

\section{Référence électronique}

Danièle Hervieu-Leger, «Le B.B. : un outil de la formation d'une communauté savante », Archives de sciences sociales des religions [En ligne], 136 | octobre - décembre 2006, mis en ligne le 02 janvier 2009, consulté le 21 avril 2019. URL : http://journals.openedition.org/assr/3815; DOI : 10.4000/assr.3815 


\section{Danièle Hervieu-Léger}

\section{Le B.B. : un outil de la formation d'une communauté savante}

La célébration d'un cinquantenaire est toujours l'occasion d'un retour sur les commencements et celui des Archives n'y échappe pas. Lorsque André Mary m'a invitée à apporter un témoignage personnel à l'événement, je me suis demandé de quelle manière je pouvais rapporter ma propre trajectoire de chercheure à celle d'une revue que j'ai commencé de fréquenter au début des années soixante-dix, et où je suis entrée moi-même en 1981 comme membre du comité de rédaction, avant que Jean Séguy me fasse l'honneur, en 1989, de me proposer à sa succession comme rédacteur en chef. Choisissant de me rapporter - de la manière la plus délibérément impressionniste - aux débuts de ce parcours, l'évocation du Bulletin bibliographique m'est apparue comme une entrée particulièrement pertinente pour évoquer le rôle de la revue dans la socialisation de la "seconde génération ", entrée dans le métier de la recherche à la suite des pionniers, fondateurs des Archives.

S'il est en effet une particularité de la Revue, dès sa fondation en 1956, c'est la place singulière qu'y occupe le Bulletin bibliographique, dit communément «B.B. ». Non qu'il soit particulièrement original, pour une revue scientifique, de donner place, en général à la fin du volume, à des recensions d'ouvrages susceptibles d'éclairer les avancées d'une discipline ou d'un champ de recherche. Ce qui frappe ici, c'est l'ampleur donnée à ce bulletin, et la présence, à la suite des comptes rendus de livres, d'un "Bulletin des périodiques » qui fit, jusqu'en 1992 (date à laquelle il fallut convenir de l'impossibilité matérielle d'accompagner l'explosion des travaux dans le domaine), état des articles significatifs parus en France ou à l'étranger.

Cette priorité accordée, dès l'origine, au suivi de la production savante consonnait, à plusieurs égards, avec le programme assigné à la revue par ses fondateurs. Pour les nouveaux venus dans le métier, le Bulletin rendait directement lisible l'ambition de ce programme. Il répondait, en premier lieu, au souci d'intéresser la revue à l'étude des faits religieux dans toute la variété de leurs manifestations, dans le temps et l'espace. Il permettait, en second lieu, de donner leur place à toute la gamme des options théoriques présentes dans le champ de 
la recherche, sans aucune exclusive. Il donnait enfin la pleine mesure de l'ouverture des Archives aux travaux publiés dans d'autres pays et dans d'autres langues. Le double Bulletin des ouvrages et des périodiques donnait ainsi à voir - au moins autant que les articles publiés dès l'origine en français ou en anglais (il faut remarquer ce dernier point qui n'avait rien d'ordinaire dans les années cinquante et soixante) - la vocation internationale de la revue et le spectre extrêmement large des travaux qu'elle se proposait d'accueillir.

Cette option imposait, par elle-même, de rassembler dans chaque numéro un volume substantiel de contributions. Celui-ci atteignit rapidement un niveau tel, et fit tant pour la réputation et l'audience de la revue, qu'il apparut tout naturel, lorsque les contraintes du routage rendirent nécessaire le passage de la publication semestrielle à la publication trimestrielle, de donner à deux des numéros une vocation entièrement bibliographique, les deux autres ayant un caractère exclusivement rédactionnel. Ce choix aurait pu être fatal à la revue du point de vue commercial et certains s'en inquiétèrent : qui en effet aurait misé sur le succès de numéros entièrement consacrés à des comptes rendus d'ouvrages et d'articles ? Ce fut pourtant le contraire qui se produisit. Certes la misère de la diffusion en librairie contribua, par défaut, à sauver ce pari éditorial plutôt risqué, en imposant à ceux qui s'intéressaient à la revue de souscrire un abonnement ! Mais les enquêtes conduites à plusieurs reprises auprès des abonnés révélèrent que le Bulletin constituait la meilleure image de marque de la revue et contribuait, de façon significative, à fidéliser son lectorat.

Ce succès - confirmé jusqu'à ce jour - n'aurait évidemment pas surpris Henri Desroche, qui plaçait expressément dans ce Bulletin - qu'il rêvait aussi exhaustif que possible - la réalisation du projet de la revue de constituer une sorte de compendium vivant de la recherche en train de se faire. Cette utopie accumulative était inscrite, selon lui, dans le titre de la revue, un titre dont lui-même revendiqua d'être l'inventeur, un jour où dans ma naïveté de jeune chercheure fraîchement entrée dans la communauté, je m'étonnais de l'austérité attachée au vocable «Archives " et suggérais d'envisager d'en changer... ${ }^{1}$.

Tout rassembler des productions d'une discipline en train de se constituer : rétrospectivement, l'entreprise paraît singulièrement peu réaliste. Mais il ne faut pas oublier que dans la seconde moitié des années cinquante et jusqu'au début des années soixante-dix, la religion ne comptait pas, à beaucoup près, parmi les objets que la sociologie, alors en plein essor, valorisait. La production nationale et internationale dans le domaine demeurait restreinte, et les chercheurs que celui-ci attirait n'étaient pas très nombreux, particulièrement en France. Ou plutôt, les institutions publiques de recherche se préoccupaient assez peu de recruter

1. Ce débat sur l'opportunité de changer le titre, considéré comme trop peu attractif, est revenu de façon récurrente au cours de l'histoire de la revue. Le changement se limita, in fine, à une mise en valeur - par le jeu des polices de caractères - des Sciences Sociales des Religions, mais sans faire disparaître la mention des Archives, devenue simplement plus discrète! 
dans ce domaine, comme en témoigna, pendant près de quinze ans après sa fondation en 1956, la composition du Groupe de Sociologie des Religions. L'évidence de la perte d'emprise des institutions religieuses, confirmée sans appel par les études empiriques de la pratique religieuse, avait sa part dans cette réticence. L'histoire religieuse, de longue tradition, demeurait florissante, mais il n'était guère mobilisateur, on peut le comprendre, de consacrer ses efforts (ou les deniers publics) à l'étude d'un objet contemporain en voie apparente de disparition. Le désintérêt portait d'ailleurs plus loin : la religion - rangée pour l'essentiel au magasin des illusions idéologiques minées par l'avancée de la science et par celle de la conscience politique des masses - ne se trouvait pas placée très haut dans l'échelle des objets légitimes de la recherche sociologique. Quant aux vocations scientifiques qu'elle suscitait, elles continuaient d'inspirer le doute ${ }^{2}$. Sauf, en effet, à s'intéresser exclusivement aux expressions passées ou exotiques du religieux, la communauté scientifique ne manquait pas de s'interroger tout bas - et même tout haut - sur les attachements personnels subreptices que révélait le choix d'étudier un objet aussi douteux.

Dans ce contexte idéologique (certes plus spécifique à la France qu'à d'autres pays), la mise à jour de l'ampleur des investissements réalisés par des chercheurs en matière d'intelligibilité proprement scientifique des faits religieux avait la vertu démonstrative et quasi-militante d'accréditer l'objet religion comme un objet intellectuel de pleine dignité. Le Bulletin fut, à coup sûr, un précieux instrument de cette habilitation académique des sciences sociales des religions à laquelle la revue tout entière contribua puissamment. Il fut aussi, par l'ampleur et la variété des travaux qu'il permettait d'approcher, un facteur précieux de la confirmation et du renforcement des vocations de chercheurs qui commençaient de s'exprimer dans un secteur de la recherche dont la génération antérieure ne leur dissimulait pas le caractère "excentré ", par rapport aux thématiques porteuses de l'époque.

Le B.B. témoignait, en particulier, de l'existence d'une recherche qui marquait avec fermeté, et même avec âpreté, son indépendance par rapport aux arrièreplans confessionnels associés à une "sociologie religieuse » dépendante de préoccupations pastorales. Il importe peu ici de savoir jusqu'à quel point ce combat contre la sociologie pastorale, fortement thématisé par les fondateurs des Archives et les animateurs du B.B., était encore réellement un enjeu de survie pour l'approche scientifique de la religion dans les dix premières années de la revue, ou s'il n'entrait pas plutôt dans la stratégie d'affirmation d'une génération - celle de l'" après Le Bras » - contrainte de faire face, dans ces mêmes années, aux conditions malaisées de l'obtention de la respectabilité sociologique pour les études sur la religion. En tout cas, le B.B. servit efficacement cette affirmation en

2. Cf. sur ce point, D. Hervieu-Léger, La religion pour mémoire, Paris, Cerf, 1993, chap. 1, La sociologie contre la religion? 
manifestant l'existence d'une communauté nationale et internationale de chercheurs en sociologie des religions dont les travaux - souffrant largement la comparaison avec ceux produits par d'autres secteurs de la discipline - témoignaient d'un rare souci d'ouverture théorique à des problématiques (durkheimiennes et surtout wébériennes) auxquelles la dominance de la vulgate marxiste d'une part, et l'envahissement de l'empirisme quantitativiste d'autre part, laissaient alors peu d'espace.

Entendons bien que cette fonction du B.B. ne fut pas seulement celle de vitrine de la discipline: l'entreprise collective que requérait sa réalisation contribua directement à constituer cette communauté de chercheurs. Le vivier des recenseurs stabilisait, autour du noyau réduit et relativement fermé des fondateurs et de ceux qui les avaient rejoints dans les toutes premières années, une population de collaborateurs réguliers, que je désignais par devers moi, à mon entrée au Groupe de Sociologie des Religions en 1974, comme les « convers », par différence avec les «moines de chœur » qui formaient le premier cercle, auquel je n'avais évidemment pas encore part!

Pour mesurer l'importance du caractère collectif de cette entreprise, du point de vue de la formation d'un milieu de recherche, il ne faut pas oublier ce qu'étaient alors les conditions du travail scientifique. L'appartement de la rue d'Athènes où la revue était fabriquée (je n'ai pas connu, pour ma part, l'époque hérö̈que des commencements du Groupe de Sociologie des Religions, rue Cardinet) n'avait en rien l'allure d'un « laboratoire » au sens que l'on donne aujourd'hui à ce terme. Les chercheurs travaillaient, pour l'essentiel, à leur domicile, chacun suivant individuellement sa voie propre, avec d'autant plus de souci de sa propre indépendance que les orientations théoriques des uns et des autres étaient différentes, et que les rivalités entre eux (des rivalités que la légende des fondations efface volontiers, mais qui ne sauraient être dissimulées s'il s'agissait d'écrire l'histoire du GSR) étaient puissantes. Certes, des entreprises collectives existaient : celle qui fit aboutir, en 1980, les travaux de géographie sociale de la pratique religieuse initiés par Fernand Boulard, poursuivis et affinés par François A. Isambert et Jean-Paul Terrenoire, en témoigne au premier chef ${ }^{3}$. Mais le Groupe de Sociologie des Religions n'en réunissait pas moins des individus qui suivaient - souvent en mobilisant les ressources d'une érudition fort impressionnante pour les chercheurs plus jeunes - des recherches disjointes, de style méthodologique et d'inspiration théorique fort disparates.

Dans ce contexte assez éclaté, le lieu collectif par excellence, le lieu où se formait l'esprit du Groupe par-delà les tensions personnelles et intellectuelles qui le traversaient, c'était la Revue. Et au sein des Archives, c'est le B.B. qui offrait l'espace relationnel le plus large, fonctionnant à la fois comme espace d'échange

3. F.A. Isambert et J.-P. Terrenoire, Atlas de la pratique religieuse des catholiques en France, Paris, FNSP/CNRS, 1980. 
entre les membres du Groupe, comme lieu de socialisation progressive (et d'évaluation!) des nouveaux entrants et, in fine, comme dispositif initiatique de l'intégration à la communauté. Se voir confier une recension dans le B.B.! Tous ceux qui sont entrés dans l'orbite du GSR à partir des années soixante-dix - la plupart du temps sous la houlette généreuse de Jean Séguy, qui fut à coup sûr le principal mentor de la génération suivante - ont fait l'expérience intimidante de la première demande de recension. Je me souviens, pour ma part, avoir sué sang et eau sur le compte rendu de l'ouvrage de Joseph Gabel Sociologie de l'aliénation, dont j'avais évoqué, avec J. Séguy justement, la pertinence pour la sociologie des religions. Je n'ai pas oublié la fierté intimidée avec laquelle j'ai reçu sa proposition d'en faire la recension. Plus qu'à mon entrée formelle au GSR, en tant qu'attachée de recherche au CNRS, c'est à cette demande que j'associe rétrospectivement mon intégration " professionnelle » au milieu des sociologues des religions.

Ce milieu prenait d'autant plus conscience de lui-même à travers la revue, et spécifiquement à travers la préparation du B.B., que la fabrication matérielle de ce dernier transformait, selon des cycles immuables, le bureau du secrétariat de la rédaction en une ruche où s'accumulaient les dactylogrammes. Commandes et envois de livres aux recenseurs, collecte des papiers, échanges avec les auteurs, relecture des épreuves, découpage minutieux des tirés à part pour l'envoi des justificatifs aux éditeurs : toutes ces étapes minutieusement enclenchées par les secrétaires de la revue étaient le moment où s'éprouvait concrètement une vie collective, par ailleurs de faible densité pour ceux des membres au moins qui n'avaient pas eu part à l'entreprise fondatrice.

C'est aussi à travers le Bulletin que j'ai commencé pour ma part, au fil des années, à percevoir quelques-uns des débats qui traversaient cette communauté. Dans cet effort de mémoire dont j'assume le caractère sélectif et subjectif, je retiendrai trois moments de discussion, touchant tous à la manière de fixer le périmètre du B.B., qui constituent, selon moi, des repères importants dans la mise à jour de la configuration intellectuelle des sciences sociales des religions, que nous pratiquons et enseignons aujourd'hui. N'ayant aucun souci ici de faire l'histoire du GSR, de la Revue ou du B.B., j'en restitue simplement la matière subjective, la façon dont ils ont, de façon plus ou moins floue, contribué à dessiner le «milieu réflexif » dans lequel un jeune chercheur fraîchement entré dans la communauté pouvait, dans les années 75-80, former sa vision personnelle du métier qu'il entendait exercer. Je me garderais, si je devais entreprendre cette histoire, de les tenir comme des clés d'interprétation du parcours de la discipline. Mais je témoigne qu'elles me permettent aujourd'hui de me rendre compte à moi-même du mien propre.

La première discussion, dont le souvenir date de mes tout premiers contacts professionnels avec les Archives, concernait l'ouverture - souhaitable ou pas du Bulletin à des ouvrages produits par des " acteurs religieux réflexifs ", prenant en charge eux-mêmes l'analyse critique des institutions religieuses et de leurs 
propres pratiques. Le débat portait principalement, mais pas exclusivement, sur la relation - à construire ou à exclure - avec la production intellectuelle du catholicisme. Cette discussion - qui s'est prolongée jusqu'au tournant des années quatre-vingt - dessinait une ligne de partage entre ceux qui mettaient en avant des exigences de clarté épistémologique incompatibles avec l'ouverture du B.B. à une littérature indigène, fût-elle critique et conceptuellement sophistiquée, et ceux qui - parfois au motif commode (et académiquement indolore) de la richesse des matériaux de recherche offerts, au second degré, par ces travaux tenaient une position, toujours sélective certes, mais moins rigoriste. Henri Desroche, fidèle à l'ambition d'un programme comparatif, interdisciplinaire et «méta-œcuménique » dans lequel il voulait faire s’unifier dialectiquement « une sociologie non religieuse de la religion et une sociologie religieuse de la non religion ", fut sans doute le seul, à l'époque de la fondation du Bulletin, à penser la possibilité que celui-ci puisse devenir aussi l'outil d'un véritable dialogue critique entre la sociologie des religions et la théologie. Je me souviens en avoir discuté avec lui en 1969 ou 1970, au moment où lui-même, constatant l'impossibilité de la réalisation de ce projet dans ce cadre, reportait cet espoir vers d'autres lieux (le centre Thomas More notamment, dont il inspira puissamment la création au couvent de l'Arbresle).

Trop lourdement parasité par le jeu des stratégies de positionnement dans le champ académique, devenu progressivement illisible pour des chercheurs plus jeunes peu au fait des conflits religieux qui avaient marqué la génération précédente, vidé enfin de sa substance par l'amenuisement d'une production théologique de haut niveau en langue française, progressivement submergé par le flot d'une littérature pastoralo-spirituelle devenue florissante, ce thème passa, au milieu des années quatre-vingt, au second plan des débats qui animaient la préparation du B.B. Il continue, rétrospectivement, de m'intéresser pour deux raisons. La première est que cette discussion - dont il faut réaliser qu'elle ne se développait pas dans les nuages, mais sur le terrain très pratique de la validation des titres retenus pour compte rendu et de la distribution des livres aux recenseurs - permettait d'introduire, de façon très concrète, une réflexion sur la manière de faire une place, dans le processus scientifique de connaissance, au sens que les acteurs eux-mêmes donnent aux situations et dynamiques historiques dans lesquelles ils s'inscrivent, ainsi qu'à l'effort critique qu'eux-mêmes déploient pour construire ce sens. La seconde raison est que l'érosion de la pointe du débat (qui ébranlait essentiellement, dans la version que je viens d'en donner, le travail de mise à distance de l'objet engagé par des chercheurs ayant souvent eux-mêmes un rapport compliqué avec le catholicisme) n'a pas fait du tout disparaître la question de la portée heuristique que revêt - en particulier pour l'analyse des processus de subjectivation qui sont au cœur de la modernité religieuse - l'exploration et la mise en perspective compréhensive de ces approches hybrides, qui conjuguent témoignage et mise à distance. Si le rapport personnel des chercheurs à leur objet 
est aujourd'hui sans doute moins convoqué dans la discussion, la difficulté des sélections à opérer pour constituer le Bulletin, de livraison en livraison, témoigne que son enjeu est toujours devant nous.

Une seconde discussion à propos du périmètre du B.B., discussion particulièrement vivante dans mon souvenir au moment où j'ai moi-même pris la responsabilité de la revue, concernait l'équilibre qu'il aurait été souhaitable d'établir, dans la masse des recensions, entre les comptes rendus des livres de sciences sociales spécifiquement et explicitement dédiés aux faits religieux, et la présentation d'ouvrages dont le propos intéressait ou croisait - à divers titres, théorique, méthodologique ou empirique - l'analyse de ces mêmes faits. Fallait-il, par exemple, "couvrir » la production de la sociologie, de la philosophie ou de l'histoire donnant une place significative à la religion dans l'analyse du pouvoir, dans l'étude des organisations, dans l'approche des logiques de la socialisation ou dans l'étude des rapports de genre ? Fallait-il élargir le spectre des ouvrages ressortissants au B.B. jusqu'à y inclure des travaux d'histoire des sciences, de science de l'éducation, de sociologie politique, de psychanalyse, etc., travaux dans lesquels le facteur religieux a une part et/ou peut ouvrir (fût-ce sur un mode analogique, comme le débat sur les « religions séculières " l'a bien montré) des voies d'interprétation suggestives? Cette autre façon de questionner la définition du périmètre du B.B. posait, de façon tout à fait pratique, la question de l'unité de la discipline et celle de la place de la sociologie des religions en son sein. Cette discussion intervenait (plus exactement: je me suis sentie concernée par elle) à un moment où un certain nombre des chercheurs qui comptaient le plus parmi les sociologues des religions s'éloignaient de leur objet premier et réorientaient leurs propres thématiques de recherche (du côté de l'éthique pour François A. Isambert ou P. Ladrière, du côté de la santé et de la médecine pour J. Maître) ${ }^{4}$. En même temps que ces déplacements témoignaient d'une tendance à un décloisonnement interne à la discipline, inséparable de la relance de l'ambition d'une sociologie générale, les questions posées par la dilatation du B.B. au-delà de la production émanant de la communauté des sociologues des religions posaient indirectement celle - fondamentale - du désenclavement des sciences sociales des religions dans le paysage général des sciences sociales. Pour opérer un tel désenclavement, il fallait à la fois sortir les travaux des spécialistes des faits religieux du confinement académique dont il a été question plus haut, intéresser d'autres secteurs des sciences sociales, et de la sociologie en particulier, à leurs apports, et élargir suffisamment la culture des uns et des autres pour rendre possible et utile l'interlocution entre eux.

On en discuta beaucoup, mais cette réflexion ne trouva pas de débouché concret réellement satisfaisant au sein du B.B., ou plutôt elle se trouva être rapidement submergée par d'autres interrogations, qui orientèrent la question de

4. Cf. F.A. IsAmbert, De la religion à l'éthique, Paris, Cerf, 1993. 
l'élargissement du B.B. dans une autre direction. La première de ces interrogations concernait la montée en puissance de la question religieuse au cœur du social, sensible dès le milieu des années soixante-dix, et devenue explosive à la fin des années quatre-vingt, avec toutes les conséquences sur la production scientifique que cette explosion impliquait. La multiplication des occurrences de la prise en compte du facteur religieux par les sciences sociales sur des terrains que la religion ne définit pas en premier lieu a eu pour effet de repousser à l'infini ou presque les efforts d' "ouverture " entrepris par les responsables du B.B. en charge des commandes de livres ${ }^{5}$. Il s'agissait moins dans ce contexte, de réfléchir sur un élargissement souhaitable que de préciser, de façon restrictive, les critères permettant de faire le tri dans le foisonnement de la production internationale. Ce qui eut pour effet de recentrer inévitablement la cible des recensions vers les ouvrages traitant spécifiquement des faits religieux.

Ce recentrage ne signifia pas pour autant un repli sur une sociologie des religions étroitement entendue. L'élargissement se fit - troisième moment des discussions sur le périmètre du B.B. - à travers une mise en valeur de plus en plus systématique de l'orientation pluridisciplinaire de la revue en général et du bulletin bibliographique en particulier. Certes, en dépit du titre que conserva la revue jusqu'en 1977, le comité de rédaction s'ouvrit très rapidement, à des spécialistes non sociologues des faits religieux : à des historiens (comme Jean Hadot, Jean-Marie Mayeur ou Jean-Pierre Vernant), à des psychologues (comme Jean-Pierre Deconchy, responsable depuis la création des Archives et jusqu'en 1982, du Bulletin des périodiques) ou à des anthropologues (comme Roger Bastide ou Jacques Guthwirth). Mais la mise en œuvre de cette dynamique interdisciplinaire, dans la construction des numéros rédactionnels à thèmes autant que dans la sélection des ouvrages, gagna nettement en densité à la fin des années quatre-vingt. C'est cet élargissement disciplinaire, en direction notamment des anthropologues et des historiens, que je me suis, pour ma part, efforcée de promouvoir pendant les années où j'ai été en charge de la revue, orientation rendue visible dans la composition d'un comité de rédaction plus nombreux, autant que dans la diversification des dossiers thématiques. Le poids croissant, à côté des recensions d'ouvrages, des « notes critiques ", formule inaugurée par Jean Séguy au moment où il était le rédacteur en chef de la revue et dont il appelait de ses vœux le développement, a été, de façon de plus en plus sensible au fil des années, un outil efficace et précieux de ce développement des interfaces interdisciplinaires sur le terrain bibliographique. Il ne fait pas de doute que cette orientation, évidemment confirmée par l'arrivée d'André Mary, lui-même anthropologue, comme

5. C'est Jean Baubérot qui prit, en 1981, le relais d'Émile Poulat, en charge du Bulletin des ouvrages depuis la création de la revue. Françoise Lautman assuma à son tour cette responsabilité, de 1987 à 1995. Éric Maigret prit sa suite en 1996 et 1997. La charge du Bulletin revint avec le $\mathrm{n}^{\circ} 100$ (en 1997) à Nathalie Luca, qui l'exerce aujourd'hui. 
rédacteur des Archives en 2004, est à mettre en relation avec le processus d'intégration d'un espace des sciences sociales des religions, qui trouve à s'épanouir notamment dans les dispositifs de l'enseignement de Master mis en place depuis 2005 sous la double responsabilité de l'École des Hautes Études en Sciences Sociales et de l'École Pratique des Hautes Études (Sciences religieuses). La revue, et le B.B., ont, à l'évidence, annoncé et anticipé ce processus.

De nouvelles interrogations s'ouvrent aujourd'hui pour la revue et le B.B., en lien avec la numérisation, la prolifération des revues en ligne, la multiplication des bases de données bibliographiques internationales, le perfectionnement des moteurs de recherche, la mise en place des pratiques nouvelles d'élaboration, de mise à disposition et de travail des textes sur des espaces numériques collaboratifs, l'expansion des archives ouvertes etc. Ces innovations technologiques, dont je me dis qu'elles auraient certainement stimulé l'imagination utopique d'Henri Desroche autant que son goût des expériences pionnières, ne modifient pas seulement - et de fond en comble - les conditions de l'échange scientifique. Elles introduisent des mutations sans précédent dans nos manières de penser, d'argumenter et de mettre en forme les connaissances. Le B.B. y survivra-t-il ? Ce n'est pas assuré. Mais on peut considérer qu'il a puissamment contribué, à travers les efforts d'exploration, de cumulation, de sélection et de hiérarchisation raisonnée des productions bibliographiques qu'il a mobilisés, à préparer une communauté de chercheurs à entrer dans ces pratiques nouvelles qui ouvrent à la recherche en sciences sociales en général, et aux sciences sociales des religions en particulier, de nouveaux horizons. 
